\title{
Chronic Eosinophilic Leukemia with FIP1L1-PDGFRA
}

National Cancer Institute

\section{Source}

National Cancer Institute. Chronic Eosinophilic Leukemia with FIP1L1-PDGFRA. NCI

Thesaurus. Code C126351.

Chronic eosinophilic leukemia characterized by the rearrangement of the PDGFRA gene, most often resulting in the formation of FIP1L1-PDGFRA fusion transcripts. 